\title{
SENSITIVITY ANALYSIS OF GENERAL MIXED MULTIVALUED MILDLY NONLINEAR VARIATIONAL INEQUALITIES
}

\author{
Salahuddin \\ Department of Mathematics \\ Aligarh Muslim University \\ Aligarh-202002, India \\ salahuddin12@mailcity.com
}

\begin{abstract}
Dafermos [1] studied the sensitivity properties of solutions of a variational inequality with respect to a parameter $\lambda$ In this paper, we extend this analysis for general mixed multivalued mildly nonlinear variational inequalities in the setting of Hilbert spaces.
\end{abstract}

Keywords- Sensitivity analysis, general mixed multivalued mildly nonlinear variational inequality, monotone and Lipschitz mappings, Hausdorff metric.

\section{INTRODUCTION}

Dafermos [1] studied the sensitivity property of solutions of a particular kind of variational inequality on a parameter which takes values on an open subset of Euclidean space $\mathrm{R}^{\mathrm{K}}$. Siddiqi et al [4], Tobin [5], Verma et al [6] studied the sensitivity analysis of various types of variational inequalities.

Let $\mathrm{H}$ be a Hilbert space and $\mathrm{D}$ be a nonempty closed and convex subset of $\mathrm{H}$.

Let $\mathrm{A}, \mathrm{T}: \mathrm{D} \rightarrow 2^{\mathrm{H}}$ be the multivalued mappings. We consider the problem of finding $\mathrm{u} \in \mathrm{D}, \mathrm{p} \in \mathrm{A}(\mathrm{u}), \mathrm{q} \in \mathrm{T}(\mathrm{u})$ such that $\mathrm{g}(\mathrm{u}) \in \mathrm{D}$

$$
\langle\mathrm{g}(\mathrm{v})-\mathrm{g}(\mathrm{u}), \mathrm{p}+\mathrm{q}\rangle+\mathrm{b}(\mathrm{u}, \mathrm{g}(\mathrm{v}))-\mathrm{b}(\mathrm{u}, \mathrm{g}(\mathrm{u})) \geq 0, \text { for all } \mathrm{g}(\mathrm{v}) \in \mathrm{D},
$$

where $b(.,):. \mathrm{H} \times \mathrm{H} \rightarrow \mathrm{R}$ satisfying the following properties:

(i) $b(.,$.$) is linear in the first argument;$

(ii) $b(.,$.$) is bounded, that is there exists constant \gamma>0$ such that

$$
|b(u, v)| \leq \gamma\|u\|\|v\| \text {, for all } u, v \in H \text {; }
$$

(iii) $|b(.,)$.$| is either convex or linear in the second argument;$

(iv) for every $\mathrm{u}, \mathrm{v}, \mathrm{w} \in \mathrm{H}$

$$
\begin{aligned}
& |b(u, v)-b(u, w)| \leq b(u, v-w) \\
& b(u, v \pm w) \leq b(u, v)+b(u, w) .
\end{aligned}
$$

In this paper, we study the sensitivity analysis of general mixed multivalued mildly nonlinear variational inequality problem (GMMMNVIP) of the type (1).

\section{SENSITIVITY ANALYSIS}

To formulate the problem, let $\mathrm{M}$ be an open subset of $\mathrm{H}$ in which the parameter $\lambda$ takes values and assume that $\left\{\mathrm{K}_{\lambda}: \lambda \in \mathrm{M}\right\}$ is a family of closed convex subset of $H$. The parametric general mixed multivalued mildly nonlinear variational inequality is to find $\mathrm{u} \in \mathrm{D},(\mathrm{p}, \lambda) \in \mathrm{A}(\mathrm{u}, \lambda),(\mathrm{q}, \lambda) \in \mathrm{T}(\mathrm{u}, \lambda)$ such that $\mathrm{g}(\mathrm{u}) \in \mathrm{K}_{\lambda}$ 


$$
\langle\mathrm{g}(\mathrm{v})-\mathrm{g}(\mathrm{u}),(\mathrm{p}, \lambda)+(\mathrm{q}, \lambda)\rangle+\mathrm{b}(\mathrm{u}, \mathrm{g}(\mathrm{v}))-\mathrm{b}(\mathrm{u}, \mathrm{g}(\mathrm{u})) \geq 0 \text {, for all } \mathrm{g}(\mathrm{v}) \in \mathrm{K}_{\lambda}
$$

where $A(u, \lambda)$ and $T(u, \lambda)$ are multivalued mappings, which are define on the set $(u, \lambda)$ with $\lambda \in \mathrm{M}$. We also assume that for some $\bar{\lambda} \in \mathrm{M}$, the problem (4) admits a solution $\bar{u}$.

We want to investigate those conditions under which, for each $\lambda$ is a neighbourhood of $\bar{\lambda}$. The problem (4) has a unique solution $u(\lambda)$ near $\bar{u}$ and the function $u(\lambda)$ is continuous and differentiable. We assume that B is the closure of a ball in $\mathrm{H}$ centered at $\pi$.

We need the following concepts.

2.1 Definition- An operator $g$ defined on $B$ to $H$ is said to be locally, $u, v \in B$,

(i) $\xi$-strongly monotone, if there exists a constant $\xi>0$ such that

$$
\langle\mathrm{g}(\mathrm{u})-\mathrm{g}(\mathrm{v}), \mathrm{u}-\mathrm{v}\rangle \geq \xi\|\mathrm{u}-\mathrm{v}\|^{2}
$$

(ii) $\sigma$-h-Lipschitz continuous, if there exists a constant $\sigma>0$ such that

$$
\|\mathrm{g}(\mathrm{u})-\mathrm{g}(\mathrm{v})\| \leq \sigma\|\mathrm{u}-\mathrm{v}\| \text {. }
$$

In particular, it follows that $\xi \leq \sigma$.

2.2 Definition- A multivalued mapping $T(u, \lambda)$ defined on $B \times M$ to $C(H)$ is said to be locally $v$-h-Lipschitz continuous, if there exists a constant $v>0$ such that

$$
\mathrm{h}(\mathrm{T}(\mathrm{u}, \lambda), \mathrm{T}(\mathrm{v}, \lambda)) \leq \mathrm{v}\|\mathrm{u}-\mathrm{v}\|, \text { for all } \mathrm{u}, \mathrm{v} \in \mathrm{B} \text {, }
$$

where $\mathrm{h}(.,$.$) is a Hausdorff metric on \mathrm{C}(\mathrm{H})$, and $\mathrm{C}(\mathrm{H})$ denotes the family of all nonempty compact subsets of $\mathrm{H}$.

2.3 Definition- A multivalued mapping $A(u, \lambda)$ defined on $B \times M$ to $C(H)$ is said to be locally $\alpha$-strongly monotone if there exists a constant $\alpha>0$ such that $\left\langle\left(\mathrm{p}_{1}, \lambda\right)-\left(\mathrm{p}_{2}, \lambda\right), \mathrm{u}-\mathrm{v}\right\rangle \geq \alpha\|\mathrm{u}-\mathrm{v}\|^{2}$ for all $\mathrm{u}, \mathrm{v} \in \mathrm{B},\left(\mathrm{p}_{1}, \lambda\right) \in \mathrm{A}(\mathrm{u}, \lambda),\left(\mathrm{p}_{2}, \lambda\right) \in \mathrm{A}(\mathrm{v}, \lambda)$.

We have the following lemmas which can be proved by the techniques of Noor [3].

2.1 Lemma- A point $u \in K_{\lambda}$ is a solution of the parametric general mixed multivalued mildly nonlinear variational inequalities (4) iff it is fixed point of the map

$$
\varphi(\mathrm{u}, \lambda)=\mathrm{u}-\mathrm{g}(\mathrm{u})+\mathrm{P}_{\mathrm{K} \lambda}[\mathrm{g}(\mathrm{u})+\mathrm{F}(\mathrm{u})-\rho((\mathrm{p}, \lambda)+(\mathrm{q}, \lambda))]
$$

for all $\lambda \in \mathrm{M},(\mathrm{p}, \lambda) \in \mathrm{A}(\mathrm{u}, \lambda),(\mathrm{q}, \lambda) \in \mathrm{T}(\mathrm{u}, \lambda)$ for some $\rho>0$, where $\mathrm{P}_{\mathrm{K} \lambda}$ is the projection of $\mathrm{H}$ on the family of closed convex sets $\mathrm{K}_{\lambda}$ and $\mathrm{F}: \mathrm{H} \rightarrow \mathrm{H}$ is a single valued function defined by, for every $(\mathrm{p}, \lambda) \in \mathrm{A}(\mathrm{u}, \lambda)$ and $(\mathrm{q}, \lambda) \in \mathrm{T}(\mathrm{u}, \lambda)$

$\langle\mathrm{F}(\mathrm{u}), \mathrm{g}(\mathrm{v})\rangle=\langle\mathrm{u}, \mathrm{g}(\mathrm{v})\rangle-\rho\langle(\mathrm{p}, \lambda)+(\mathrm{q}, \lambda), \mathrm{g}(\mathrm{v})\rangle-\rho \mathrm{b}(\mathrm{u}, \mathrm{g}(\mathrm{v}))$, for all $\mathrm{g}(\mathrm{v}) \in \mathrm{K}_{\lambda}$.

Since we are interested in the case, when the solution of the problem (4) lies in the interior of B. So we consider the map $\varphi^{*}(\mathrm{u}, \lambda)$ define by

$\varphi^{*}(\mathrm{u}, \lambda)=\mathrm{u}-\mathrm{g}(\mathrm{u})+\mathrm{P}_{\mathrm{K \lambda лв}}[\mathrm{g}(\mathrm{u})+\mathrm{F}(\mathrm{u})-\rho((\mathrm{p}, \lambda)+(\mathrm{q}, \lambda))]$, for all $(\mathrm{u}, \lambda) \in \mathrm{B} \times \mathrm{M}$.

We have to show that the map $\varphi^{*}(\mathrm{u}, \lambda)$ has a fixed point, which by $(9)$ is also a solution of (4). First of all, we prove that the map $\varphi^{*}(u, \lambda)$ is a contraction map with respect to $u$, uniformly in $\lambda \in \mathrm{M}$, by using locally $\alpha$-strongly monotonicity and locally $\beta$-h-Lipschitz continuity of the operator $A(u, \lambda)$, locally $\xi$-strongly monotonicity and $\sigma$-h-Lipschitz continuity of $\mathrm{g}(\mathrm{u})$ and locally $\mathrm{v}$-h-Lipschitz continuity of $\mathrm{T}(\mathrm{u}, \lambda)$.

2.2 Lemma- For all $u_{1}, u_{2} \in B$ and $\lambda \in M$, we have

$$
\left\|\varphi^{*}\left(\mathrm{u}_{1}, \lambda\right)-\varphi^{*}\left(\mathrm{u}_{2}, \lambda\right)\right\| \leq \theta\left\|\mathrm{u}_{1}-\mathrm{u}_{2}\right\|
$$

where

$$
\theta=\mathrm{k}+2 \rho v+\rho \gamma+2\left(1-2 \rho \alpha+\rho^{2} \beta^{2}\right)^{1 / 2}
$$

for

$$
((1-\mathrm{k})(2 v+\gamma)) 4^{-1}<\alpha<\beta, \quad \mathrm{k}<1,
$$


and

$$
\alpha>(1-\mathrm{k})(\gamma+2 v) 4^{-1}+\left(\left(\beta^{2}-\left((2 v+\gamma) 2^{-1}\right)^{2}\right)(3-\mathrm{k})(1+\mathrm{k}) 4^{-1}\right)^{1 / 2}
$$

$\left|\rho-\left(\alpha+(\mathrm{k}-1)(\gamma+2 v) 4^{-1}\right)\left(\beta^{2}-\left((2 v+\gamma) 2^{-1}\right)^{2}\right)^{-1}\right|<$

$\left.\left\{\left(\alpha+(\mathrm{k}-1)(\gamma+2 v) 4^{-1}\right)^{2}-\left(\beta^{2}-\left((2 v+\gamma) 2^{-1}\right)^{2}\right)(1+\mathrm{k})(3-\mathrm{k}) 4^{-1}\right)\right\}^{1 / 2}\left\{\beta^{2}-\left((2 v+\gamma) 2^{-1}\right)^{2}\right\}^{-1}$ with

Proof- Using (11), we have

$$
\mathrm{k}=2\left(1-2 \xi+\sigma^{2}\right)^{1 / 2} \text {. }
$$

$\left\|\varphi^{*}\left(\mathrm{u}_{1}, \lambda\right)-\varphi^{*}\left(\mathrm{u}_{2}, \lambda\right)\right\|=\|\left\{\mathrm{u}_{1}-\mathrm{g}\left(\mathrm{u}_{1}\right)+\mathrm{P}_{\mathrm{K} \lambda}\left[\mathrm{g}\left(\mathrm{u}_{1}\right)+\mathrm{F}\left(\mathrm{u}_{1}\right)-\rho\left(\left(\mathrm{p}_{1}, \lambda\right)+\left(\mathrm{q}_{1}, \lambda\right)\right)\right]\right\}$

$$
-\left\{\mathrm{u}_{2}-\mathrm{g}\left(\mathrm{u}_{2}\right)+\mathrm{P}_{\mathrm{K} \lambda}\left[\mathrm{g}\left(\mathrm{u}_{2}\right)+\mathrm{F}\left(\mathrm{u}_{2}\right)-\rho\left(\left(\mathrm{p}_{2}, \lambda\right)+\left(\mathrm{q}_{2}, \lambda\right)\right)\right]\right\} \|
$$

and using the fact that the projection operator is nonexpansive, we have

$$
\begin{array}{r}
\left\|\varphi^{*}\left(\mathrm{u}_{1}, \lambda\right)-\varphi^{*}\left(\mathrm{u}_{2}, \lambda\right)\right\| \leq 2\left\|\mathrm{u}_{1}-\mathrm{u}_{2}-\left(\mathrm{g}\left(\mathrm{u}_{1}\right)-\mathrm{g}\left(\mathrm{u}_{2}\right)\right)\right\|+\left\|\mathrm{F}\left(\mathrm{u}_{1}\right)-\mathrm{F}\left(\mathrm{u}_{2}\right)\right\| \\
+\left\|\mathrm{u}_{1}-\mathrm{u}_{2}-\rho\left(\left(\mathrm{p}_{1}, \lambda\right)-\left(\mathrm{p}_{2}, \lambda\right)\right)\right\|+\rho\left\|\left(\mathrm{q}_{1}, \lambda\right)-\left(\mathrm{q}_{2}, \lambda\right)\right\| .
\end{array}
$$

Now, the operator $\mathrm{g}(\mathrm{u})$ is both locally $\xi$-strongly monotone and $\sigma$-h-Lipschitz continuous and the operator $\mathrm{A}(\mathrm{u}, \lambda)$ is locally $\alpha$-strongly monotone and locally $\beta$-hLipschitz continuous, so by the method of Noor [2],

$$
\begin{aligned}
\left\|\mathrm{u}_{1}-\mathrm{u}_{2}-\left(\mathrm{g}\left(\mathrm{u}_{1}\right)-\mathrm{g}\left(\mathrm{u}_{2}\right)\right)\right\|^{2} & =\left\|\mathrm{u}_{1}-\mathrm{u}_{2}\right\|^{2}+\left\|\mathrm{g}\left(\mathrm{u}_{1}\right)-\mathrm{g}\left(\mathrm{u}_{2}\right)\right\|^{2}-2\left\langle\mathrm{~g}\left(\mathrm{u}_{1}\right)-\mathrm{g}\left(\mathrm{u}_{2}\right), \mathrm{u}_{1}-\mathrm{u}_{2}\right\rangle \\
& \leq\left(1-2 \xi+\sigma^{2}\right)\left\|\mathrm{u}_{1}-\mathrm{u}_{2}\right\|^{2}
\end{aligned}
$$

where $\sigma$ and $\xi$ are Lipschitz and strongly monotone constants of $g(u)$, respectively and

$$
\begin{gathered}
\left\langle\mathrm{F}\left(\mathrm{u}_{1}\right)-\mathrm{F}\left(\mathrm{u}_{2}\right), \mathrm{g}(\mathrm{v})\right\rangle= \\
-\left\langle\mathrm{u}_{1}-\mathrm{u}_{2}, \mathrm{~g}(\mathrm{v})\right\rangle-\rho\left\langle\left(\mathrm{p}_{1}, \lambda\right)-\left(\mathrm{p}_{2}, \lambda\right), \mathrm{g}(\mathrm{v})\right\rangle \\
-\rho\left\langle\left(\mathrm{q}_{1}, \lambda\right)-\left(\mathrm{q}_{2}, \lambda\right), \mathrm{g}(\mathrm{v})\right\rangle-\rho \mathrm{b}\left(\mathrm{u}_{1}-\mathrm{u}_{2}, \mathrm{~g}(\mathrm{v})\right) \\
\leq\left\{\left\|\mathrm{u}_{1}-\mathrm{u}_{2}-\rho\left(\left(\mathrm{p}_{1}, \lambda\right)-\left(\mathrm{p}_{2}, \lambda\right)\right)\right\|+\rho\left\|\left(\mathrm{q}_{1}, \lambda\right)-\left(\mathrm{q}_{2}, \lambda\right)\right\|+\rho \gamma\left\|\mathrm{u}_{1}-\mathrm{u}_{2}\right\|\right\}\|\mathrm{g}(\mathrm{v})\| \\
\leq\left\{\left(1-2 \alpha \rho+\rho^{2} \beta^{2}\right)^{1 / 2}+\rho v+\rho \gamma\right\}\left\|\mathrm{u}_{1}-\mathrm{u}_{2}\right\|\|\mathrm{g}(\mathrm{v})\| .
\end{gathered}
$$

Now

$\left\|\mathrm{F}\left(\mathrm{u}_{1}\right)-\mathrm{F}\left(\mathrm{u}_{2}\right)\right\|=\sup _{\mathrm{g}(\mathrm{v}) \in \mathrm{K}}\left\{\left\langle\mathrm{F}\left(\mathrm{u}_{1}\right)-\mathrm{F}\left(\mathrm{u}_{2}\right), \mathrm{g}(\mathrm{v})\right\rangle /\|\mathrm{g}(\mathrm{v})\|\right\}$

and

$$
\leq\left\{\left(1-2 \alpha \rho+\rho^{2} \beta^{2}\right)^{1 / 2}+\rho v+\rho \gamma\right\}\left\|\mathrm{u}_{1}-\mathrm{u}_{2}\right\|
$$

$\left\|\mathrm{u}_{1}-\mathrm{u}_{2}-\rho\left(\left(\mathrm{p}_{1}, \lambda\right)-\left(\mathrm{p}_{2}, \lambda\right)\right)\right\|^{2}=\left\|\mathrm{u}_{1}-\mathrm{u}_{2}\right\|^{2}+\rho^{2}\left\|\left(\mathrm{p}_{1}, \lambda\right)-\left(\mathrm{p}_{2}, \lambda\right)\right\|^{2}$

Using (6) and (8), we have

$$
-2 \rho\left\langle u_{1}-u_{2},\left(p_{1}, \lambda\right)-\left(p_{2}, \lambda\right)\right\rangle \text {. }
$$

$$
\begin{gathered}
\left\|\left(\mathrm{p}_{1}, \lambda\right)-\left(\mathrm{p}_{2}, \lambda\right)\right\| \leq \mathrm{h}\left(\mathrm{A}\left(\mathrm{u}_{1}, \lambda\right), \mathrm{A}\left(\mathrm{u}_{2}, \lambda\right)\right) \leq \beta\left\|\mathrm{u}_{1}-\mathrm{u}_{2}\right\| \\
\left\langle\mathrm{u}_{1}-\mathrm{u}_{2},\left(\mathrm{p}_{1}, \lambda\right)-\left(\mathrm{p}_{2}, \lambda\right)\right\rangle \geq \alpha\left\|\mathrm{u}_{1}-\mathrm{u}_{2}\right\|^{2} .
\end{gathered}
$$

Therefore (15) becomes

$$
\begin{aligned}
\left\|\mathrm{u}_{1}-\mathrm{u}_{2}-\rho\left(\left(\mathrm{p}_{1}, \lambda\right)-\left(\mathrm{p}_{2}, \lambda\right)\right)\right\|^{2} & \leq\left\|\mathrm{u}_{1}-\mathrm{u}_{2}\right\|^{2}+\rho^{2} \beta^{2}\left\|\mathrm{u}_{1}-\mathrm{u}_{2}\right\|^{2}-2 \rho \alpha\left\|\mathrm{u}_{1}-\mathrm{u}_{2}\right\|^{2} \\
& \leq\left(1-2 \alpha \rho+\rho^{2} \beta^{2}\right)\left\|\mathrm{u}_{1}-\mathrm{u}_{2}\right\|^{2}
\end{aligned}
$$


where $\beta$ and $\alpha$ are the constants appearing in (5), (6), again

$$
\left\|\left(\mathrm{q}_{1}, \lambda\right)-\left(\mathrm{q}_{2}, \lambda\right)\right\| \leq \mathrm{h}\left(\mathrm{T}\left(\mathrm{u}_{1}, \lambda\right), \mathrm{T}\left(\mathrm{u}_{2}, \lambda\right)\right) \leq \mathrm{v}\left\|\mathrm{u}_{1}-\mathrm{u}_{2}\right\| .
$$

Since $T(u, \lambda)$ is locally $v$-h-Lipschitz continuous, using (13)-(17), we have

$$
\left\|\varphi^{*}\left(\mathrm{u}_{1}, \lambda\right)-\varphi^{*}\left(\mathrm{u}_{2}, \lambda\right)\right\| \leq \theta\left\|\mathrm{u}_{1}-\mathrm{u}_{2}\right\|
$$

where

$$
\theta=\mathrm{k}+2 \rho v+\rho \gamma+2\left(1-2 \rho \alpha+\rho^{2} \beta^{2}\right)^{1 / 2}
$$

with

$$
\mathrm{k}=2\left(1-2 \xi+\sigma^{2}\right)^{1 / 2} \text {. }
$$

Now, using the technique of Noor [3], we can show that $\theta<1$ from which it follows that the map $\varphi^{*}(\mathrm{u}, \lambda)$ defined by $(11)$ is a contraction map.

2.1 Remark- From Lemma 2.2, It is clear that the map $\varphi^{*}(\mathrm{u}, \lambda)$ defined by $(11)$ has a unique fixed point $\mathrm{u}(\lambda)$ that is $\mathrm{u}(\lambda)=\varphi^{*}(\mathrm{u}, \lambda)$, we also know by assumption, the function $\overline{\mathrm{u}}$ for $\lambda=\bar{\lambda}$ is a solution of the parametric general mixed multivalued mildly nonlinear variational inequality problem (4), we see that $\bar{u}$ is a fixed point of $\varphi(u, \lambda)$ and it is also a fixed point of $\varphi^{*}(\mathrm{u}, \lambda)$, consequently we have $\mathrm{u}(\bar{\lambda})=\bar{u}=\varphi^{*}(\mathrm{u}(\bar{\lambda}), \bar{\lambda})$, now we show that the solution $u(\lambda)$ of problem (4) is $\beta$-h-Lipschitz continuous.

2.3 Lemma- If $\mathrm{T}(\overline{\mathrm{u}}, \lambda), \mathrm{A}(\overline{\mathrm{u}}, \lambda)$ is the multivalued operators and the map

$$
\left.\left.\lambda \rightarrow \mathrm{P}_{\mathrm{K} \lambda \cap \mathrm{B}}[\mathrm{g}(\overline{\mathrm{u}})+\mathrm{F}(\overline{\mathrm{u}})-\rho(\overline{(\mathrm{p}, \lambda})+\overline{(\mathrm{q}}, \bar{\lambda})\right)\right]
$$

are $\beta$-h- Lipschitz continuous in $\lambda$ at $\lambda$, then $u(\lambda)$ is $\beta$-h- Lipschitz continuous at $\lambda=\lambda$.

Proof- Fix $\lambda \in \mathrm{M}$, then using the triangle inequality and Lemma 2.2, we have

$$
\begin{aligned}
& \|\mathrm{u}(\lambda)-\mathrm{u}(\bar{\lambda})\| \leq\left\|\varphi^{*}(\mathrm{u}(\lambda), \lambda)-\varphi^{*}(\mathrm{u}(\bar{\lambda}), \lambda)\right\|+\left\|\varphi^{*}(\mathrm{u}(\bar{\lambda}), \lambda)-\varphi^{*}(\bar{u}(\bar{\lambda}), \bar{\lambda})\right\| \\
& \leq \theta\|\mathrm{u}(\lambda)-\mathrm{u}(\bar{\lambda})\|+\left\|\varphi^{*}(\mathrm{u}(\bar{\lambda}), \lambda)-\varphi^{*}(\overline{\mathrm{u}} \overline{(\bar{\lambda})}, \bar{\lambda})\right\| .
\end{aligned}
$$

From (11) and the fact that the projection operator is nonexpansive, we have

$$
\begin{aligned}
& \left.\left.\left\|\varphi^{*}(\mathrm{u}(\bar{\lambda}), \lambda)-\varphi^{*}(\mathrm{u}(\bar{\lambda}), \bar{\lambda})\right\|=\| \mathrm{P}_{\mathrm{K} \text { лпв }}[\mathrm{g}(\mathrm{u}(\bar{\lambda}))+\mathrm{F}(\mathrm{u}(\bar{\lambda}))-\rho((\mathrm{p} \overline{(\lambda)}), \lambda)+(\mathrm{q} \overline{(\bar{\lambda}}), \lambda)\right)\right] \\
& -\mathrm{P}_{\mathrm{K} \wedge \text { в }}[\mathrm{g}(\mathrm{u}(\bar{\lambda}))+\mathrm{F}(\mathrm{u}(\bar{\lambda}))-\rho((\mathrm{p}(\bar{\lambda}), \bar{\lambda})+(\mathrm{q}(\bar{\lambda}), \bar{\lambda}))] \| \\
& \leq \rho\|(\mathrm{p}(\bar{\lambda}), \lambda)-(\mathrm{p}(\bar{\lambda}), \bar{\lambda})\|+\rho\|(\mathrm{q}(\bar{\lambda}), \lambda)-(\mathrm{q}(\bar{\lambda}), \bar{\lambda})\| \\
& \begin{array}{c}
+\| \mathrm{P}_{\mathrm{K} \lambda \mathrm{B}}[\mathrm{g}(\mathrm{u}(\bar{\lambda}))+\mathrm{F}(\overline{\mathrm{u}(\bar{\lambda})})-\rho((\mathrm{p}(\bar{\lambda}), \bar{\lambda})+(\mathrm{q}(\bar{\lambda}), \bar{\lambda}))] \\
-\mathrm{P}_{\mathrm{K}} \overline{\lambda त \mathrm{~B}}_{\mathrm{B}}[\mathrm{g}(\overline{\mathrm{u}(\bar{\lambda}))}+\mathrm{F}(\overline{\mathrm{u}(\bar{\lambda}))}-\rho((\mathrm{p}(\bar{\lambda}), \bar{\lambda})+(\mathrm{q}(\bar{\lambda}), \bar{\lambda}))] \| .
\end{array}
\end{aligned}
$$

Now from Remark 2.1 and combining (18) and (19), we have

$$
\begin{aligned}
& \|\mathrm{u}(\lambda)-\overline{\mathrm{u}}\| \leq \rho(1-\theta)^{-1}\|(\mathrm{p}(\lambda), \lambda)-(\mathrm{p}(\bar{\lambda}), \bar{\lambda})\|+\rho(1-\theta)^{-1}\|(\mathrm{q}(\lambda), \lambda)-(\mathrm{q}(\bar{\lambda}), \bar{\lambda})\| \\
& \left.\left.+\rho(1-\theta)^{-1} \| P_{K} \text { तกB }[g(\bar{u})+F(\bar{u})-\rho((p \bar{\lambda}), \bar{\lambda})+(q \bar{\lambda}), \bar{\lambda})\right)\right]
\end{aligned}
$$




$$
-\mathrm{P}_{\mathrm{K}} \overline{\lambda \cap \mathrm{B}}[\mathrm{g}(\overline{\mathrm{u}})+\mathrm{F}(\overline{\mathrm{u}})-\rho((\mathrm{p}(\bar{\lambda}), \bar{\lambda})+(\mathrm{q}(\bar{\lambda}), \bar{\lambda}))] \| .
$$

From which the required result follows:

Using the technique of Dafermos [1], we can show that there exists a neighbourhood $\mathrm{N}$ containing in $M$ of $\bar{\lambda}$ such that for $\lambda \in N, u(\lambda)$ is the unique solution of problem (4) in the interior of $\mathrm{B}$.

Combining the above results we arrive at the following:

2.1 Theorem- Let $\bar{u}$ be the solution of parametric general mixed multivalued mildly nonlinear variational inequality problem (4) at $\lambda=\lambda$, the multivalued mappingT(u, $\lambda$ ) be locally $v$-h-Lipschitz continuous and the multivalued mapping $A(\bar{u}, \lambda)$ be locally $\alpha$ strongly monotone and locally $\beta$-h-Lipschitz continuous, the map $\mathrm{g}(\overline{\mathrm{u}})$ be locally $\xi$ strongly monotone and $\sigma$-h-Lipschitz continuous.

Suppose that $\mathrm{T} \overline{(\mathrm{u}}, \lambda), \mathrm{A}(\overline{\mathrm{u}}, \lambda), \mathrm{g} \overline{\mathrm{u}})$ and the map

$$
\left.\left.\lambda \rightarrow \mathrm{P}_{\mathrm{K} \lambda \mathrm{B} B}[\mathrm{~g} \overline{(\mathrm{u})}+\overline{\mathrm{F}(\mathrm{u})}-\rho \overline{(\mathrm{p}}, \bar{\lambda})+\overline{(\mathrm{q}}, \overline{\lambda)}\right)\right]
$$

are $\beta$-h-Lipschitz continuous at $\lambda=\bar{\lambda}$, then there exists a neighbourhood $\mathrm{N}$ containing in $\mathrm{M}$ of $\lambda$ such that for $\lambda \in \mathrm{N}$, the problem (4) has a unique solution $\mathrm{u}(\lambda)$ in the interior of $\mathrm{B}, \mathrm{u}(\bar{\lambda})=\overline{\mathrm{u}}$ and $\mathrm{u}(\lambda)$ are continuous $(\beta$-h-Lipschitz continuous) at $\lambda=\bar{\lambda}$.

2.2 Remark- The function $u(\lambda)$ as defined in Theorem 2.1 is continuously differentiable on some neighbourhood $N$ of $\lambda$. For this see Dafermos [1].

\section{REFERENCES}

1. S. Dafermos, Sensitivity analysis in variational inequalities, Math.Oper. Res. 13, 421434, (1988).

2. M. A. Noor, An iterative scheme for a class of quasivariational inequalities, J. Math. Anal. Appl. 110, 463-468, (1985).

3. M. A. Noor, On a class of variational inequalities, J. Math. Anal. Appl. 128, 138155, (1987).

4. A. H. Siddiqi and R. Ahmad, On generalized complementarity problem, Math. Comput. Appl. 2(1), 31-43, (1997).

5. R. L. Tobin, Sensitivity analysis for variational inequalities, J. Optim. Theo. Appl. 48, 191-204, (1986).

6. H. L. Verma and R. N. Mukherjee, Sensitivity analysis of generalized variational inequalities, J. Math. Anal. Appl. 167, 299-304, (1992). 